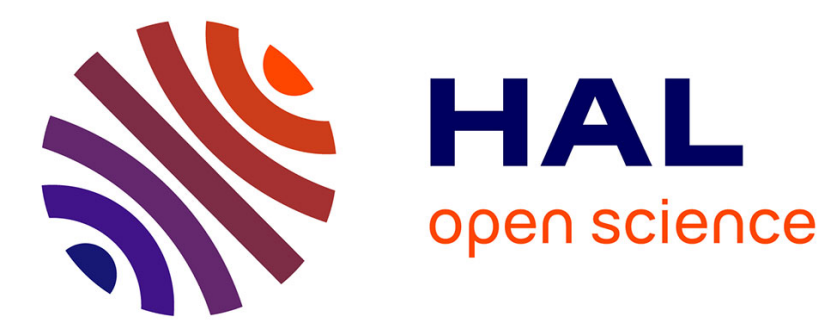

\title{
Numerical simulation of flows past flat plates using volume penalization
}

Kai Schneider, Mickaël Paget-Goy, Alberto Verga, Marie Farge

\section{To cite this version:}

Kai Schneider, Mickaël Paget-Goy, Alberto Verga, Marie Farge. Numerical simulation of flows past flat plates using volume penalization. Computational \& Applied Mathematics, 2014, 33 (2), pp.481-495. 10.1007/s40314-013-0076-9 . hal-01500522

\section{HAL Id: hal-01500522 \\ https://hal-amu.archives-ouvertes.fr/hal-01500522}

Submitted on 6 Apr 2017

HAL is a multi-disciplinary open access archive for the deposit and dissemination of scientific research documents, whether they are published or not. The documents may come from teaching and research institutions in France or abroad, or from public or private research centers.
L'archive ouverte pluridisciplinaire HAL, est destinée au dépôt et à la diffusion de documents scientifiques de niveau recherche, publiés ou non, émanant des établissements d'enseignement et de recherche français ou étrangers, des laboratoires publics ou privés. 


\section{Numerical simulation of flows past flat plates using volume penalization}

\section{Kai Schneider, Mickaël Paget-Goy, Alberto Verga \& Marie Farge}

Computational and Applied Mathematics

ISSN 0101-8205

Comp. Appl. Math.

DOI 10.1007/s40314-013-0076-9
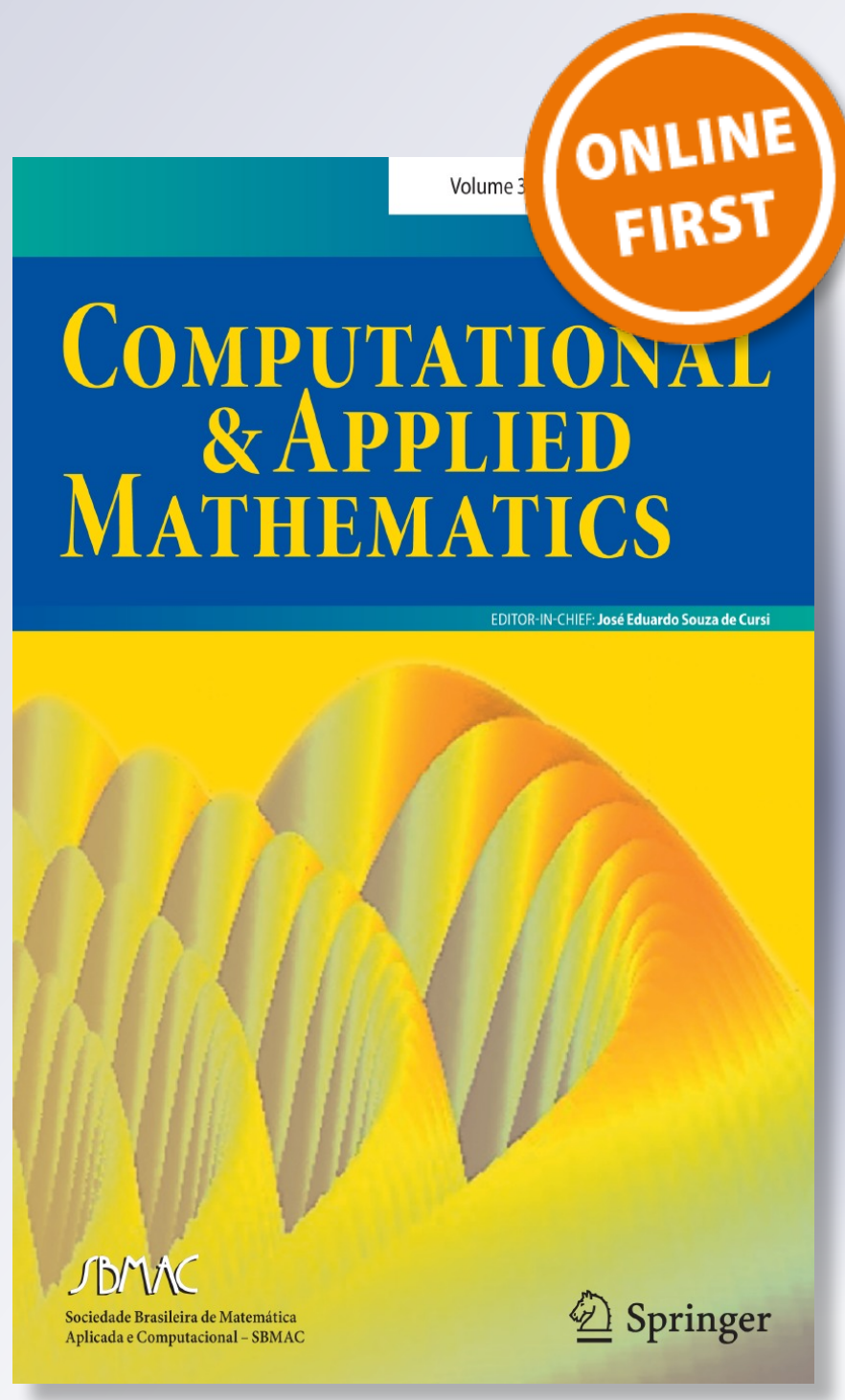

焦 Springer 
Your article is protected by copyright and all rights are held exclusively by SBMAC Sociedade Brasileira de Matemática Aplicada e Computacional. This e-offprint is for personal use only and shall not be self-archived in electronic repositories. If you wish to self-archive your article, please use the accepted manuscript version for posting on your own website. You may further deposit the accepted manuscript version in any repository, provided it is only made publicly available 12 months after official publication or later and provided acknowledgement is given to the original source of publication and a link is inserted to the published article on Springer's website. The link must be accompanied by the following text: "The final publication is available at link.springer.com". 


\title{
Numerical simulation of flows past flat plates using volume penalization
}

\author{
Kai Schneider • Mickaël Paget-Goy • \\ Alberto Verga - Marie Farge
}

Received: 22 December 2012 / Revised: 29 April 2013 / Accepted: 12 September 2013

(C) SBMAC - Sociedade Brasileira de Matemática Aplicada e Computacional 2014

\begin{abstract}
We present numerical simulations of two-dimensional viscous incompressible flows past flat plates having different kind of wedges: one tip of the plate is rectangular, while the other tip is either a wedge with an angle of $30^{\circ}$ or a round shape. We study the shear layer instability of the flow considering different scenarios, either an impulsively started plate or an uniformly accelerated plate, for Reynolds number $R e=9500$. The volume penalization method, with either a Fourier spectral or a wavelet discretization, is used to model the plate geometry with no-slip boundary conditions, where the geometry of the plate is simply described by a mask function. On both tips, we observe the formation of thin shear layers which are rolling up into spirals and form two primary vortices. The self-similar scaling of the spirals corresponds to the theoretical predictions of Saffman for the inviscid case. At later times, these vortices are advected downstream and the free shear layers undergo a secondary instability. We show that their formation and subsequent dynamics is highly sensitive to the shape of the tips. Finally, we also check the influence of a small riblet, added on the back of the plate on the flow evolution.
\end{abstract}

Communicated by Eduardo Souza de Cursi.

K. Schneider $(\varangle)$

M2P2-CNRS, Aix-Marseille Université, 38 rue F. Joliot-Curie,

13541 Marseille Cedex 20, France

e-mail:kschneid@cmi.univ-mrs.fr

M. Paget-Goy

M2P2-CNRS, Aix-Marseille Université, 38 rue F. Joliot-Curie,

13451 Marseille Cedex 20, France

A. Verga

IM2NP-CNRS, Aix-Marseille Université, Avenue Escadrille Normandie Niemen,

Case 142, 13397 Marseille Cedex 20, France

M. Farge

LMD-CNRS, Ecole Normale Supérieure, 24 rue Lhomond, 75231 Paris Cedex 05, France

Published online: 08 February 2014

基 springer $S \mathrm{D} / \mathrm{MAC}$ 
Keywords Instability of shear layers - Vortex dynamics - Computational methods in fluid dynamics $\cdot$ Free shear layers $\cdot$ Wavelets $\cdot$ Spectral methods

Mathematics Subject Classification (2000) Primary 65M85; Secondary 76D17 - 65T60 * $65 \mathrm{M} 70$

\section{Introduction}

Flow past a thin flat plate moving normal to the free stream has been subject to many experimental, theoretical and numerical investigations (Pierce 1961; Saffman 1995; Koumoutsakos and Shiels 1996). When the plate is impulsively started from rest (relatively to the flow), a primary spiral vortex forms and develops at the edges of the plate. At later times, when these start-up vortices are advected downstream, the formation of secondary vortices along the primary vortex sheet are observed, for sufficiently large Reynolds numbers.

The study of the start-up vortex can be traced back to the work on 'Fluid motion at very small viscosity' of Prandtl (1905) who analyzed its initial evolution and showed that it can be described by a class of self-similar solutions of the Euler equations.

The stability of the start-up vortex was questioned by several authors. Moore (1976) proved the stability of certain stretching spiral vortices. Pullin (1978) performed numerical simulations of self-similar spiral start up vortices and noted that Moore's demonstration did not fully apply to them. Luchini and Tognaccini $(1999,2002)$ computed inviscid vortex shedding from a flat plate and observed that the spiral vortex exhibits oscillations due to a secondary instability. Their results were in qualitative agreement with predictions made for inviscid flows.

Different laboratory experiments have also been performed (Pierce 1961; Pullin and Perry 1980). Pullin and Perry (1980) studied the spiral vortices at an earlier stage, in which viscous effects are comparable with convective ones, whereas Pierce's (1961) results explored later stage, in which a mainly inviscid flow has become unstable. Higuchi et al. (1996) computed the time history of circulation and studied the onset of three-dimensional vortex shedding in the wake behind a disk. The experimental part of their study showed that the secondary instability developed for both uniformly accelerated and for impulsively started flat plate. Koumoutsakos and Shiels (1996) computed with a vortex method the flow normal to a plate, which is either uniformly accelerated or impulsively started. They observed the secondary instability only for the accelerated case. Nitsche (1996) applied a vortex sheet model to the formation of a vortex ring at the edge of a circular tube. Wang et al. (1999) computed, using compact finite differences of fourth order, the flow normal to a thin ellipse. They found the formation of secondary vortices for the impulsively started case at high enough Reynolds number $(R e=10000)$. Their observation is in agreement with the experiment of Pierce (1961). They also proposed to relate the presence of small corner vortices, adjacent to the tips of the plate and induced by the primary vortices, to the formation of secondary vortices. Pullin and Perry (1980) explained the secondary vortices by a shear layer instability. The stability of the vortex sheet roll-up and its secondary instability has also been studied by Abid and Verga (2002).

By means of direct numerical simulation (DNS) we study the flow normal to a flat plate, either impulsively started or accelerated. A suitable approach for this task is the penalization method which has been introduced by Arquis and Caltagirone (1984). Therewith, walls or solid obstacles are modeled as a porous medium whose porosity $\eta$ tends to zero. The Navier-Stokes equations are modified accordingly by adding a Darcy term. Fluid regions are 
considered as completely permeable, while regions where walls or obstacles are present as perfectly impermeable. The geometry of the flow is simply taken into account by a spatially varying permeability coefficient, which enables an easy practical implementation of the method and allows to consider more complex obstacles and situations, e.g., moving obstacles and fluid-structure interaction. A mathematical theory proving convergence of this physically based approach has been given by Angot et al. (1999). The penalization method has been applied in the context of low order methods (finite difference/volume schemes, e.g., Khadra et al. 2000; Angot et al. 1999), using pseudospectral methods, e.g., Farge and Schneider (2001), Kevlahan and Ghidaglia (2001), Schneider (2005), Schneider and Farge (2005), Keetels et al. (2007) and also adaptive wavelet methods (Schneider and Farge 2002; Keetels et al. 2007). The latter scheme automatically adapts the spatial grid, not only to the flow evolution, but also to the geometry of walls or bluff bodies (Schneider and Farge 2002). For a recent review on wavelet methods in computational fluid dynamics, we refer to Schneider and Vasilyev (2010).

In the present paper, we apply the penalization method using, as numerical scheme, a Fourier pseudo-spectral method and in one case an adaptive wavelet method, to study the flow past a flat plate moving normal to the free stream. First, we investigate the self-similar roll-up of the starting vortex and check the scaling of the spiral's radius with Kaden's theoretical prediction (Saffman 1995), which depends on the geometry of the plate and on the shape of the tip. Then, we revisit the formation of secondary instabilities for both uniformly accelerated and impulsively started plate, together with the dependence of the secondary vortex formation on the shape of the tip, i.e., for wedge, rectangular and round shape. Finally, we investigate the influence of the corner vortex on the secondary instability by modifying the geometry of the plate. For this we add a very small riblet on the lee-side of the plate.

At later times, when the primary vortices are advected downstream, we observe the formation of secondary vortices along the primary vortex sheets, for sufficiently large Reynolds numbers. These results agree with observations in laboratory experiments (Pierce 1961) and with other numerical simulations using a vortex method (Koumoutsakos and Shiels 1996).

The paper is organised as follows: first, we present the penalization method together with the numerical schemes used to solve the penalized Navier-Stokes equations numerically. As application, we present numerical simulations of two-dimensional viscous incompressible flow past a flat plate, which is either impulsively started normal to the free stream at $R e=$ 9500 , or uniformly accelerated. We also modify the plate's geometry by adding a small riblet to study its influence on the formation of the secondary vortices. Finally, we give some conclusions and perspectives for turbulence modeling of bluff body flows.

\section{The penalization method and the numerical discretisation}

\subsection{Governing equations}

The penalization technique is based on the physical idea which consists in modeling solid walls or obstacles as porous media whose porosity $\eta$ tends to zero (Arquis and Caltagirone 1984). The geometry is described by a mask function $\chi(\vec{x})$, which is 1 inside the solid regions and 0 elsewhere. Note that the penalization method can also take into account obstacles with time-varying shape by simply introducing a time-dependent mask function (Kolomenskiy and Schneider 2009). The Navier-Stokes equations are modified by adding a supplementary term containing the mask function. For the 'penalized' velocity $\vec{u}_{\eta}$, we obtain

$$
\partial_{t} \vec{u}_{\eta}+\vec{u}_{\eta} \cdot \nabla \vec{u}_{\eta}+\nabla p_{\eta}-v \nabla^{2} \vec{u}_{\eta}+\frac{1}{\eta} \chi_{\Omega_{s}}\left(\vec{u}_{\eta}-\vec{u}_{p}(t)\right)=0
$$




$$
\nabla \cdot \vec{u}_{\eta}=0
$$

where $\vec{u}_{\eta}(\vec{x}, t)$ is the flow velocity, $p_{\eta}(\vec{x}, t)$ the pressure, $\vec{u}_{p}(t)$ the obstacle's velocity, and $v$ the kinematic viscosity. The mask function is given by

$$
\chi_{\Omega_{s}}(\vec{x})= \begin{cases}1 & \text { for } \vec{x} \\ 0 & \text { elsewhere }\end{cases}
$$

where $\Omega_{s}$ denotes the solid obstacle. The equations (2.1) and (2.2) are solved in the domain $\Omega$, which consists of both the fluid and the solid domain imposing periodic boundary conditions at the boundary of $\Omega$. For $\eta \longrightarrow 0$, the flow evolution is governed by the Navier-Stokes equations in the fluid regions, and by Darcy's law, i.e., the velocity is proportional to the pressure gradient, in the solid regions where obstacles or walls are present. In Carbou and Fabrie (2003), a mathematical proof has been given that the above equations converge towards the Navier-Stokes equations with no-slip boundary conditions, with order $\eta^{1 / 2}$, in the limit $\eta$ tending to zero, which improves the previous estimate (Angot et al. 1999). In numerical simulations, an improved convergence of order $\eta$ has been reported.

The resulting forces $\vec{F}$ on the obstacle, i.e., drag and lift, can be computed by integrating the penalized velocity over the obstacle's volume (Angot et al. 1999):

$$
\begin{aligned}
\vec{F} & =\lim _{\eta \rightarrow 0} \int_{\Omega_{s}} \nabla p_{\eta} \mathrm{d} x=-\lim _{\eta \rightarrow 0} \frac{1}{\eta} \int_{\Omega_{s}} \vec{u}_{\eta} \mathrm{d} x \\
& =\int_{\partial \Omega_{s}} \sigma(\vec{u}, p) \cdot \vec{n} \mathrm{~d} \gamma
\end{aligned}
$$

where $\Omega_{s}$ is the obstacle's volume, $\partial \Omega_{s}$ its boundary, $\vec{n}$ its outer normal and $\sigma(\vec{u}, p)=$ $\frac{v}{2}\left(\nabla \vec{u}+(\nabla \vec{u})^{t}\right)-p I$ the stress tensor. Hence, the lift and drag forces on the obstacle, i.e., forces parallel and perpendicular to the free-stream velocity of the flow, are easy to compute as volume integrals instead of contour integrals.

For two-dimensional flows the vorticity-velocity formulation is prefered, and therefore we take the curl of Eq. (2.1), and we get

$$
\begin{array}{r}
\partial_{t} \omega_{\eta}+\left(\vec{u}_{\eta}+\vec{U}_{\infty}\right) \cdot \nabla \omega_{\eta}-v \nabla^{2} \omega_{\eta} \\
+\nabla \times\left(\frac{1}{\eta} \chi_{\Omega_{s}}\left(\vec{u}_{\eta}\right)-\vec{u}_{p}(t)\right)=0
\end{array}
$$

where $\omega=\nabla \times \vec{u}$ is the vorticity and $\vec{U} \infty$ is the free-stream velocity, a constant mean flow which is the only potential flow for periodic boundary conditions.

\subsection{Numerical methods}

For the numerical solution of the penalized equations we employ, a classical Fourier pseudospectral method (Canuto et al. 1988; Kevlahan and Ghidaglia 2001; Schneider 2005), and in one case also a wavelet scheme to illustrate the possibility of adaptive grid refinement (Fröhlich and Schneider 1997; Schneider and Farge 2002). A benchmark and a thorough validation of both methods applied to a dipole-wall collision can be found in Keetels et al. (2007). 


\subsubsection{Fourier pseudospectral method}

Fourier pseudospectral discretizations are classical schemes in CFD, which are highly accurate for flows with periodic boundary conditions. For a more complete discussion, we refer the reader to Canuto et al. (1988). Equation (2.5) is transformed into Fourier space to compute the spatial derivatives and to evolve the vorticity field in time. Terms containing products, i.e., the convection and penalization terms, are calculated by collocation in physical space. Hence, the vorticity field and the other variables are represented as truncated Fourier series

$$
\omega(\vec{x}, t)=\sum_{\vec{k} \in \mathbb{Z}^{2}} \widehat{\omega}(\vec{k}, t) \exp (i \vec{k} \cdot \vec{x})
$$

where the Fourier transform of $\omega$ is defined as

$$
\widehat{\omega}(\vec{k}, t)=\frac{1}{4 \pi^{2}} \int \omega(\vec{x}, t) \exp (-i \vec{k} \cdot \vec{x}) \mathrm{d} \vec{x}
$$

with the wavevector $\vec{k}=\left(k_{x}, k_{y}\right)$. The Fourier discretization is uniform in space and is truncated at $k_{x}=-N_{x} / 2$ and $k_{x}=N_{x} / 2-1, k_{y}=-N_{y} / 2$ and $k_{y}=N_{y} / 2-1$, where $N_{x}$ and $N_{y}$ are the number of grid points in $x$ and $y$ direction, respectively. The gradient of $\omega$ is computed by multiplying $\widehat{\omega}$ with $i \vec{k}$, the Laplacian by multiplying with $|\vec{k}|^{2}$. The velocity $\vec{u}$ induced by the vorticity $\omega$ is reconstructed in Fourier space using Biot-Savart's law

$$
\vec{u}(\vec{x}, t)=\sum_{\vec{k} \in \mathbb{Z}^{2}, \vec{k} \neq 0} \frac{i \vec{k} \perp}{|\vec{k}|^{2}} \widehat{\omega}(\vec{k}, t) \exp (i \vec{k} \cdot \vec{x}),
$$

where $\vec{k} \perp=\left(-k_{y}, k_{x}\right)$.

The convection term $\vec{u} \cdot \nabla \omega$ and the penalization term $\nabla \times\left(\frac{1}{\eta} \chi_{\Omega_{s}}\left(\vec{u}-\vec{u}_{p}(t)\right)\right.$ are evaluated by the pseudospectral technique using collocation in physical space. To avoid aliasing errors, i.e., the production of small scales due to the nonlinear terms which are not resolved on the grid, we de-aliase at each time step, by truncating the Fourier coefficients using the $2 / 3$ rule,

$$
\widehat{\omega}(\vec{k})=\left\{\begin{array}{lll}
\widehat{\omega}(\vec{k}) & \text { for } & \left(\frac{3 k_{x}}{2 N_{x}}\right)^{2}+\left(\frac{3 k_{y}}{2 N_{y}}\right)^{2}<1, \\
0 & \text { for } & \left(\frac{3 k_{x}}{2 N_{x}}\right)^{2}+\left(\frac{3 k_{y}}{2 N_{y}}\right)^{2} \geq 1
\end{array}\right.
$$

For the transformation between physical and Fourier space we use Temperton's Fast Fourier Transform, which has a complexity of order $\mathbf{N} \log _{2} \mathbf{N}$, with $\left(\mathbf{N}=N_{x} N_{y}\right)$ (Canuto et al. 1988).

For the time discretization, we use a semi-implicit scheme with adaptive time-stepping (Schneider 2005). The linear diffusion term is discretized implicitly using exact time integration which is cheap for spectral methods, as the Laplace operator is diagonal in Fourier space, and hence no linear system has to be solved. This improves the stability limit of purely explicit schemes. The remaining terms are discretized explicitly using second-order AdamsBashforth extrapolation. This avoids the solution of nonlinear equations, however it implies a CFL condition on the maximum size of the time step.

The step size control of the time step is based on the CFL stability limit of the explicit discretization of the nonlinear term. Therefore in each time step $t_{n}$, pointwise the maximal rms velocity is computed, 


$$
u_{\max }=\max _{\vec{x}} \sqrt{|\vec{u}(\vec{x})|^{2}}
$$

and the new time step is given by

$$
\Delta t_{n+1}=C \Delta x / u_{\max }
$$

with the minimal spatial grid size $\Delta x=\min \left(\frac{L_{x}}{N_{x}}, \frac{L_{y}}{N_{y}}\right)$, where $L_{x}$ and $L_{y}$ denote the length of the domain in $x$ and $y$ direction, respectively, and $C<1$ is the CFL constant.

In addition $\Delta t$ has to be smaller than $\eta$, which is the stability limit imposed by the explicit discretization of the penalization term.

\subsubsection{Adaptive wavelet method}

As adaptive schemes dynamically adapt the spatial grid at each time step, we first discretize the equation (2.5) in time using semi-implicit finite differences, i.e., Euler-backwards for the viscous term and Adams-Bashforth extrapolation for the nonlinear term, which are both of second order (Peyret 2002).

The resulting elliptic problem to be solved at each time step is:

$$
\begin{aligned}
\left(\gamma I-v \nabla^{2}\right) \omega^{n+1}= & \frac{4}{3} \gamma \omega^{n}-\frac{1}{3} \gamma \omega^{n-1}-\nabla \cdot\left(\omega^{\star}\left(\vec{u}^{\star}+\vec{U}_{\infty}\right)\right) \\
& -\nabla \times\left(\frac{1}{\eta} \chi\left(\vec{u}^{\star}-\vec{u}_{p}\right)\right)
\end{aligned}
$$

where

$$
\omega^{\star}=2 \omega^{n}-\omega^{n-1} \quad \vec{u}^{\star}=2 \vec{u}^{n}-\vec{u}^{n-1}
$$

with time step $\Delta t, \gamma=3 /(2 \Delta t)$ and $I$ representing the identity.

For the space discretization we use a Petrov-Galerkin scheme. Therefore, the vorticity is developed into a set of trial functions, and the minimization of the weighted residual of (2.11) requires that the projection onto a space of test functions vanishes. As space of trial functions, we employ a two-dimensional multiresolution analysis (MRA) (Farge 1992) and develop $\omega^{n}$ at time step $n$ into an orthonormal wavelet series

$$
\omega^{n}(x, y)=\sum_{\lambda}\left\langle\omega^{n}, \psi_{\lambda}\right\rangle \psi_{\lambda}(x, y)
$$

with the multi-index $\lambda=\left(j, i_{x}, i_{y}, \mu\right)$, where $j=0, J_{\max }-1$ denotes the scale $2^{-j+1},\left(i_{x}, i_{y}\right)=0, \ldots, 2^{j}-1$ the position and $\mu=1,2,3$ the three different directions of the wavelets. The inner product is defined by $\langle f, g\rangle=\int f(x, y) g(x, y) d x d y$.

The test functions $\theta_{\lambda}$ are defined as solutions of the linear part of Eq. (2.11)

$$
\left(\gamma I-v \nabla^{2}\right) \theta_{\lambda}=\psi_{\lambda}
$$

and can be computed in a preprocessing step. This avoids assembling the stiffness matrix and solving a linear equation at each time step. The functions $\theta$, called vaguelettes, are explicitly calculated in Fourier space and have similar localization properties as wavelets (Fröhlich 
and Schneider 1997). The solution of (2.11) in wavelet space therewith reduces to a change of basis:

$$
\begin{aligned}
\tilde{\omega}_{\lambda}= & \left\langle\omega^{n+1}, \psi_{\lambda}\right\rangle \\
= & \left\langle\left(\frac{4}{3} \gamma \omega^{n}-\frac{1}{3} \gamma \omega^{n-1}-\nabla \cdot\left(\omega^{\star}\left(\vec{u}^{\star}+\vec{U}_{\infty}\right)\right)\right.\right. \\
& \left.\left.-\nabla \times\left(\frac{1}{\eta} \chi\left(\vec{u}^{\star}-\vec{u}_{p}\right)\right)\right), \theta_{\lambda}\right\rangle .
\end{aligned}
$$

Nonlinear wavelet thresholding is applied in each time step to obtain an adaptive discretization by retaining only those wavelet coefficients $\tilde{\omega}_{\lambda}$ with absolute value above a given threshold $\epsilon=\epsilon_{0} \sqrt{Z}$, where $\epsilon_{0}$ is a constant and $Z=\frac{1}{2} \int|\omega(\vec{x})|^{2} \mathrm{~d} \vec{x}$ is the enstrophy. For the next time step, the index coefficient set (which addresses each coefficient in wavelet space) is determined by adding neighbours to the retained wavelet coefficients. Consequently, only those coefficients $\tilde{\omega}$ in (2.15) belonging to this extrapolated index set are computed using the adaptive vaguelette decomposition (Fröhlich and Schneider 1997). The nonlinear term $-\nabla \cdot\left(\omega^{\star}\left(\vec{u}^{\star}+\vec{U} \infty\right)\right)-\nabla \times\left(\frac{1}{\eta} \chi\left(\vec{u}^{\star}-\vec{u}_{p}\right)\right)$ is evaluated by partial collocation on a locally refined grid (Schneider and Farge 2002). The vorticity $\omega^{\star}$ is reconstructed in physical space on an adaptive grid from its wavelet coefficients $\tilde{\omega}^{\star}$, using the adaptive wavelet reconstruction algorithm (Fröhlich and Schneider 1997). From the adaptive vaguelette decomposition replacing $\theta$ in Eq. (2.14) by $\theta=\left(\nabla^{2}\right)^{-1} \psi$, we solve $\nabla^{2} \Psi^{\star}=\omega^{\star}$ to get the stream function $\tilde{\Psi}^{\star}$ and reconstruct $\Psi^{\star}$ on a locally refined grid. By means of centered finite differences of fourth order, we compute $\nabla \omega^{\star}, \vec{u}^{\star}=\left(-\partial_{y} \Psi^{\star}, \partial_{x} \Psi^{\star}\right)$ and $\nabla \times\left(\frac{1}{\eta} \chi\left(\vec{u}^{\star}-\vec{u}_{p}\right)\right)$ on the adaptive grid. Subsequently, the nonlinear term is summed up pointwise, and finally (2.15) is solved using the adaptive vaguelette decomposition.

\section{Numerical results}

\subsection{Flow configuration}

We study the early evolution of incompressible viscous flows past a flat plate which is displaced in its normal direction (Fig. 1), either impulsively started, or uniformly accelerated. We have chosen one tip of the plate to be rectangular, while the other is a wedge with an angle of $30^{\circ}$ (cf. Fig. 1). The two-dimensional approximation, we use here, remains valid, since we focus on the non-stationary flow behavior at early times. At $t=0$, the plate is either impulsively started

$$
\vec{u}_{p}(t)= \begin{cases}u_{0} & t \geq 0 \\ 0 & t<0\end{cases}
$$

with velocity $u_{0}=1$, or uniformly accelerated with velocity

$$
\vec{u}_{p}(t)= \begin{cases}a t & t \geq 0 \\ 0 & t<0\end{cases}
$$

and with the acceleration $a=1$. We define a Reynolds number $R e=\frac{u_{p} L}{v}$ based on the length of the plate $L$, which evolves in time for the uniformly accelerated case. 


\subsection{Spiral formation at early times}

The flow past a flat plate has been studied theoretically as potential flow in the complex plane (Saffman 1995). Following Saffman (1995), a Kutta condition is imposed at the tip of the plate by adding a spiral vortex to the potential flow (see Fig. 2). Saffman (1995) predicted a self-similar scaling of the spiral, which depends on the angle of the wedge $\alpha$ and the acceleration law of the plate.

We perform several numerical simulations with resolution $N_{x}=N_{y}=2048$ and $\eta=$ $10^{-4}$ using the Fourier pseudo-spectral method, except in Fig. (3) where the adaptive wavelet method has been used (with $\epsilon_{0}=10^{-3}$ ) to illustrate the grid adaptation shown in Fig. 4. Figure 3 shows the vorticity field at $t=1.4$ for the impulsively started plate at $R e=9500$. The flow is characterized by the roll-up of the two free shear layers produced at the tips into

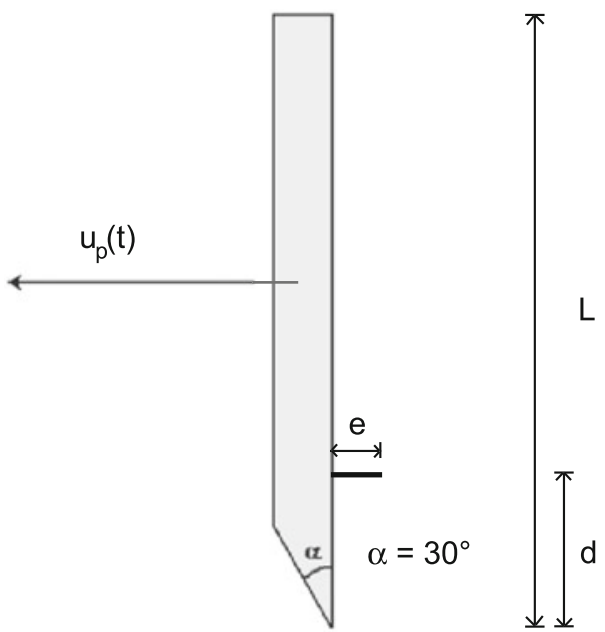

Fig. 1 Geometry of the flat plate and direction of its motion

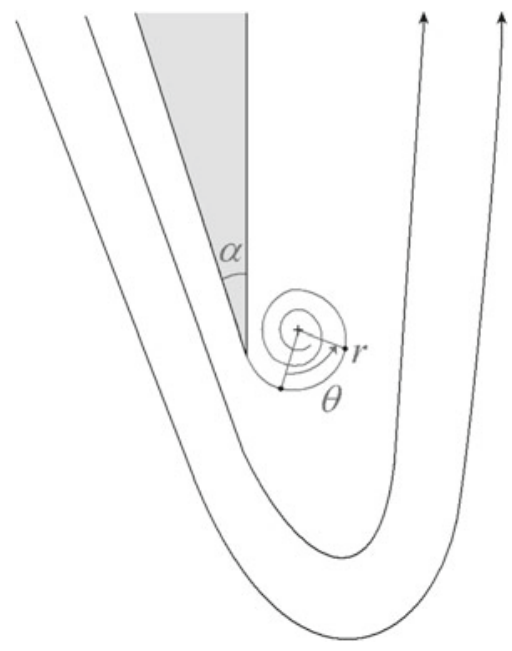

Fig. 2 Sketch of the flow configuration 


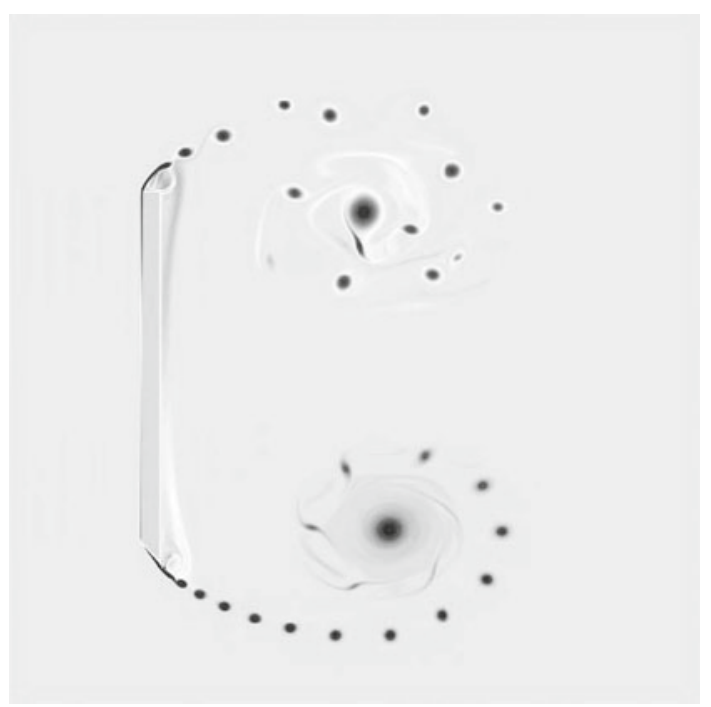

Fig. 3 Vorticity field (zoom) at $t=1.4$ for the impulsively started plate at $R e=9500$

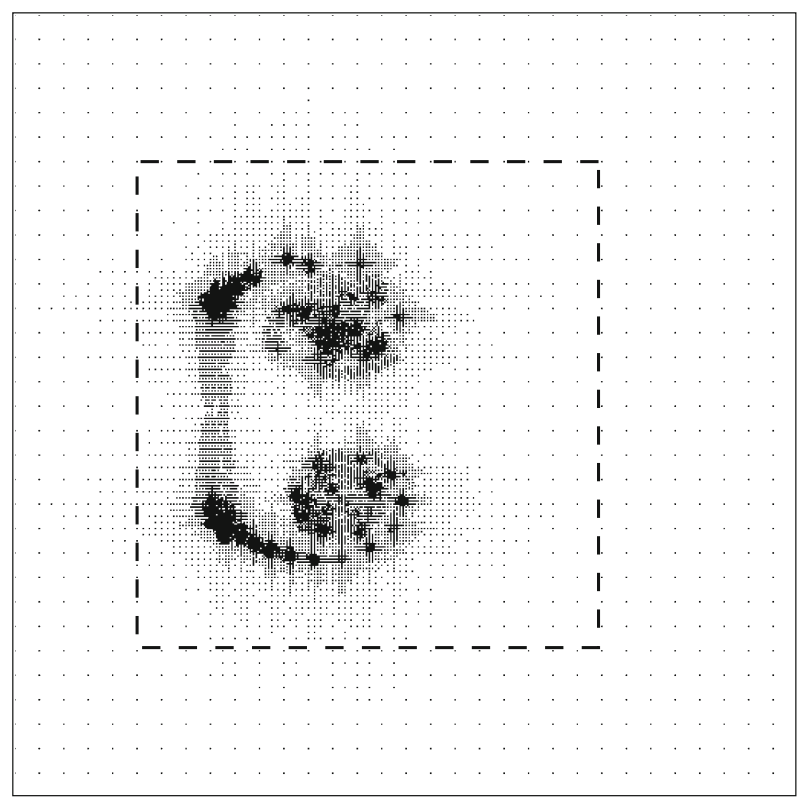

Fig. 4 Corresponding adaptive grid (computational domain) at $t=1.4$ for the impulsively started plate at $R e=9500$. The dashed square indicates the domain of the vorticity field shown in Fig. 3

two counter-rotating primary vortices developing behind the plate. The two resulting large scale recirculation zones create two boundary layers on the back of the plate, separated by a stagnation point located in the middle.

These boundary layers produce vorticity, of opposite sign to the primary vortices, and form a corner vortex at each tip of the plate. Figure 3 also exhibits secondary vortices resulting from an instability of the two shear layers. We observe that the shape of the tips (square 


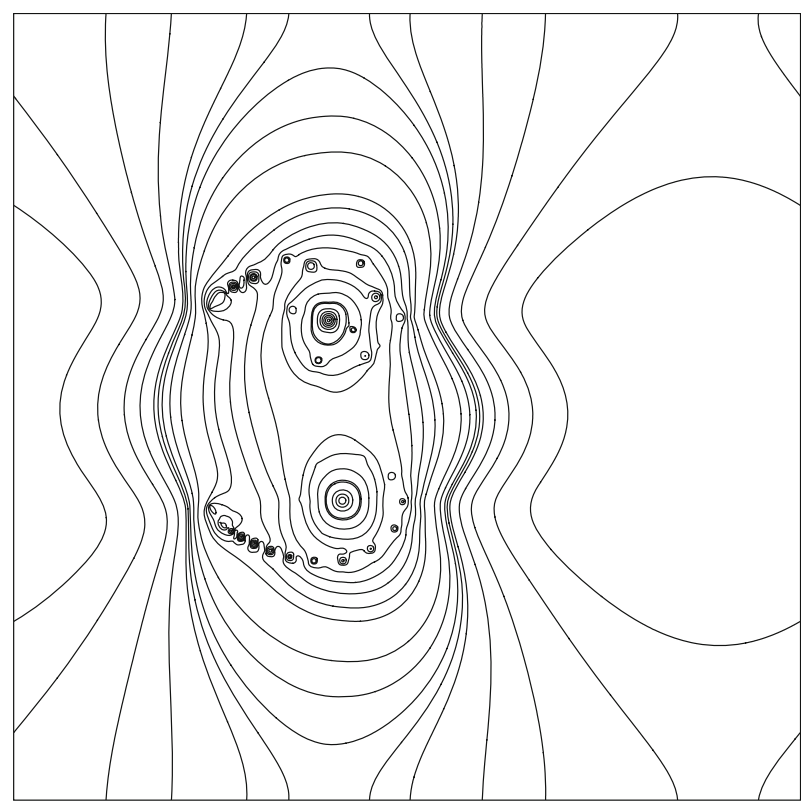

Fig. 5 Isocontours of pressure $(p=-6: 0 @ 0.75)$ at $t=1.4$ for the impulsively started plate at $R e=9500$

shape on the top, and wedge on the bottom) strongly influences this secondary instability, since the spatial distribution of the secondary vortices differs significantly. For the wedge (bottom) the distance between two subsequent vortices is continuously increasing, from the edge to the center of the primary vortex, where they disappear under the straining of the latter. In contrast, we observe for the square edge (top) an irregular distribution of the secondary vortices, whose strength remains sufficient to resist the straining of the primary vortex, and hence they penetrate further towards its center. The corresponding adaptive grid (Fig. 4) of the wavelet computation shows that grid points are locally refined in regions of strong gradients of vorticity.

The pressure can be computed from the velocity by solving a Poisson equation with periodic boundary conditions,

$$
\nabla^{2} p=-\nabla(\vec{u} \cdot \nabla \vec{u})-\frac{1}{\eta} \nabla \cdot\left(\chi_{\Omega_{s}}\left(\vec{u}_{\eta}-\vec{u}_{p}(t)\right)\right)
$$

The isocontours of pressure, shown in Fig. 5, nicely illustrate the centers of the vortices by circular well-pronounced low pressure contours.

In the following, we focus on the lower half of the plate with a wedge of $\alpha=30^{\circ}$ and study the formation of the primary vortex.

At early times, we observe the formation of a thin vortex sheet which is rolling up into a spiral and forms the primary vortex (Fig. 8, top). Figure 6 shows a zoom of the spiral (case $\alpha=30^{\circ}$ ) at early time for the impulsively started plate $(t=0.4)$ and the uniformly accelerated plate $(t=0.8)$, i.e., we plot vorticity isolines to study the scaling law of the spiral vortex and to check Saffman's predictions Saffman (1995). The coordinates of the spiral $r(\theta)$ with respect to its center are obtained by analyzing the isolines of the vorticity fields in Fig. 6. In Fig. 7, we plot the scaling of the spiral's radius for both, the impulsively started and the uniformly accelerated case in double logarithmic representation. For both, 
Fig. 6 Zoom of the primary vortex spiral at $t=0.4$ for an impulsively started plate at $R e=9500$ (left) and at $t=0.8$ for an uniformly accelerated plate (right). Isolines of vorticity $(-100,-10$ by step of 2$)$
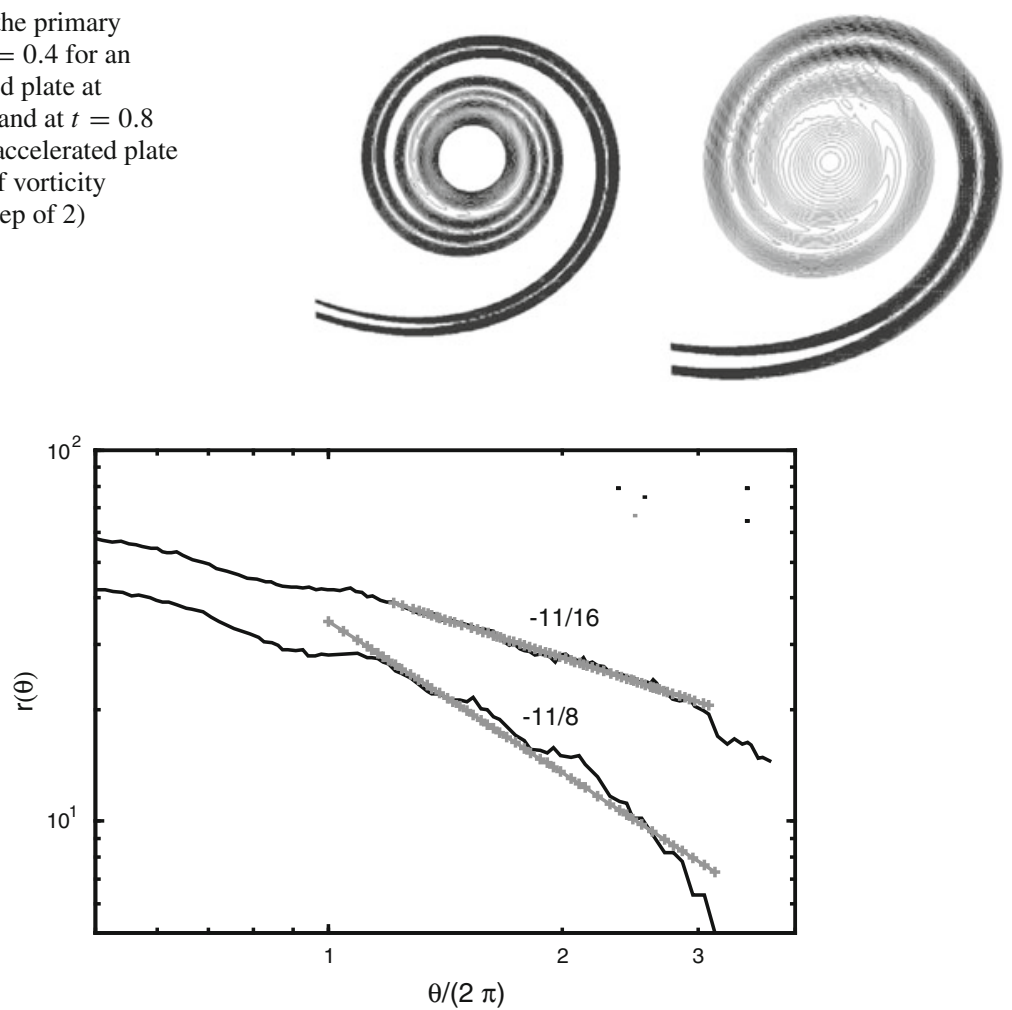

Fig. 7 Scaling of the spiral's radius versus the angle $\theta$ for both the impulsively started (line with slope $-11 / 16)$ and for the uniformly accelerated case (line with slope $-11 / 8$ )

we observe a self-similar behavior with a scaling of $r(\theta) \propto \theta^{-11 / 16}$ for the former, and of $r(\theta) \propto \theta^{-11 / 8}$ for the latter. Applying linear regression to the $\log -\log$ representation of $r(\theta)$, excluding the points of the first turn, we find indeed a slope of -0.6875 for the impulsively started case and of -1.3574 for the uniformly accelerated case. Both values agree reasonably well with the theoretical predictions of $-11 / 16$ and $-11 / 8$, respectively. Saffman (1995) predicted these values for the inviscid case, i.e., for infinite Reynolds numbers, concerning the roll up of vortex sheets for an accelerated flow past a wedge with the same angle. The impulsively started flow corresponds to the limit case of an infinite acceleration and is also well predicted.

\subsection{Formation of secondary vortices at later times}

The two primary vortices formed on the upper and lower tip are advected downstream. For sufficiently large Reynolds numbers $(R e>5000)$, we observe that the shear layers undergo a secondary instability at later times. This instability leads to the formation of secondary vortices along the primary vortex sheets (Figs. 3, 8), as observed in both laboratory and numerical experiments at similar Reynolds numbers (Koumoutsakos and Shiels 1996; Pierce 1961; Wang et al. 1999).

We also observe that the formation of the secondary vortices and their dynamics is highly sensitive to the shape of the tips. To study this, we consider two different shapes for the edges 

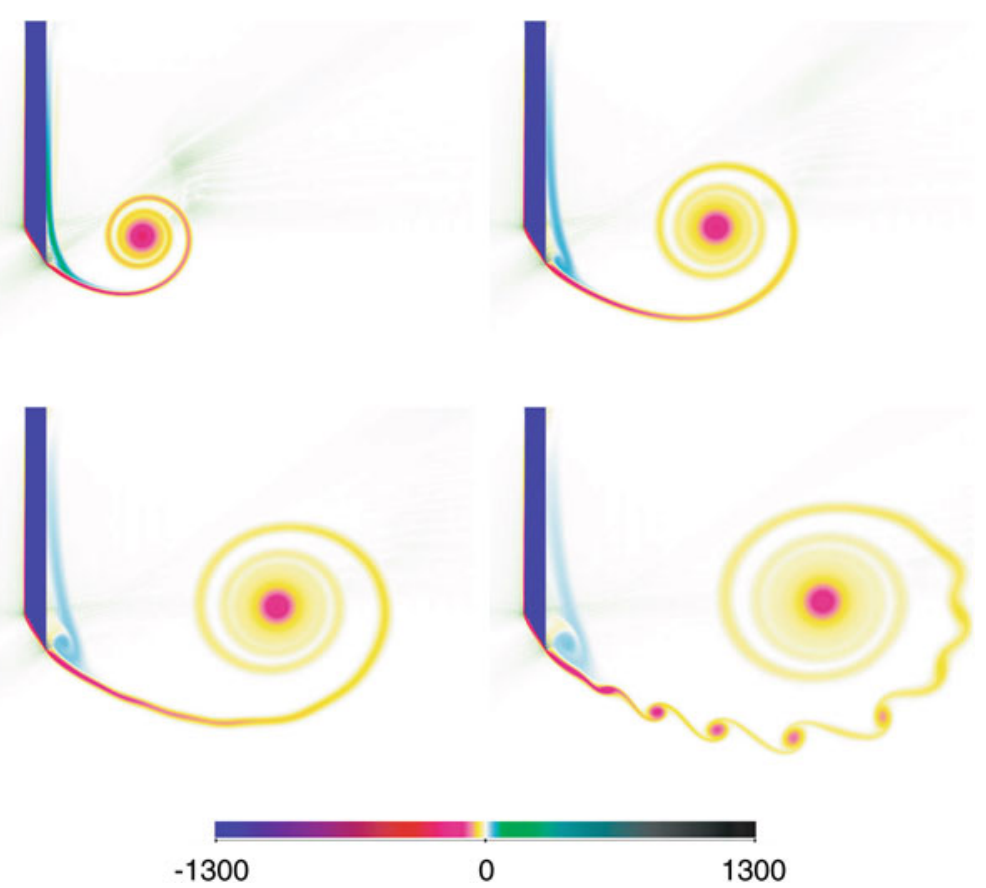

0

1300

Fig. 8 Flow past an impulsively started plate with a sharp wedge of angle $\alpha=30^{\circ}$ at $\operatorname{Re}=9500$. Vorticity fields at $t=0.39,0.75,1.11$ and 1.47
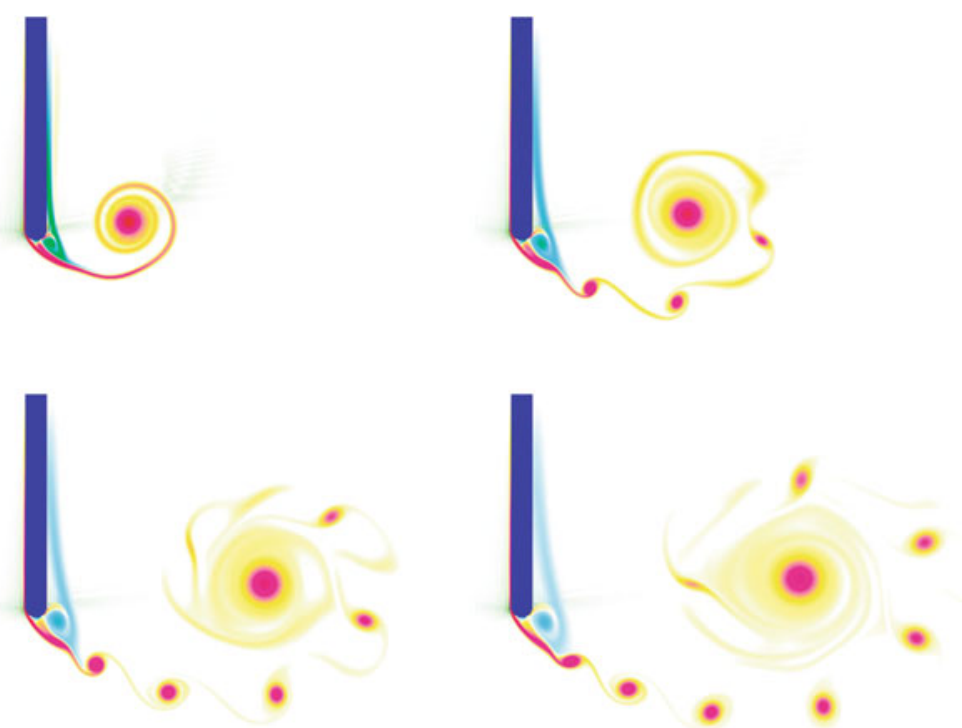

Fig. 9 Flow past an impulsively started plate with a round edge at $R e=9500$. Vorticity at $t=0.39,0.75,1.11$ and 1.47

Q2 Springer $\mathscr{D} \mathfrak{M} M \mathbb{A C}$ 
of the plate: a wedge with angle $\alpha=30^{\circ}$ and a round edge. We compare the corresponding flow evolution, and we find that the formation of the secondary instability is delayed in the case of the wedge; i.e., at $t=0.75$ no secondary vortices are observed (Fig. 8, top, right), while they are already well formed in the case of the round edge (Fig. 9, top, right). For a square edge (not shown here), we have observed that the secondary instability occurs even slightly earlier than for the round edge. This suggests that the position of the separation point seems to control the onset of this instability, since it is displaced depending on the shape of the edge: from the left for the square, towards the middle for the round, to the right for the wedge. Correspondingly, the position of the counter-rotating corner vortex is displaced likewise, from the left to the right, which suggests that it may trigger the secondary instability. In Wang et al. (1999) it has been conjectured that the periodicity of the secondary vortex formation corresponds to the rotation frequency of the corner vortex. In order to control the intensity of the corner vortex, and therefore its rotation frequency, we added a riblet on the back of the plate. As the riblet is in the recirculation zone behind the plate (cf. Fig. 1), it produces vorticity of the same sign as the corner vortex. We thus control its intensity, by varying the position $d$ and the size $e$ of the riblet, and performed several numerical experiments to check the riblet's influence on the secondary instability. In Fig. 10, we show the vorticity field at
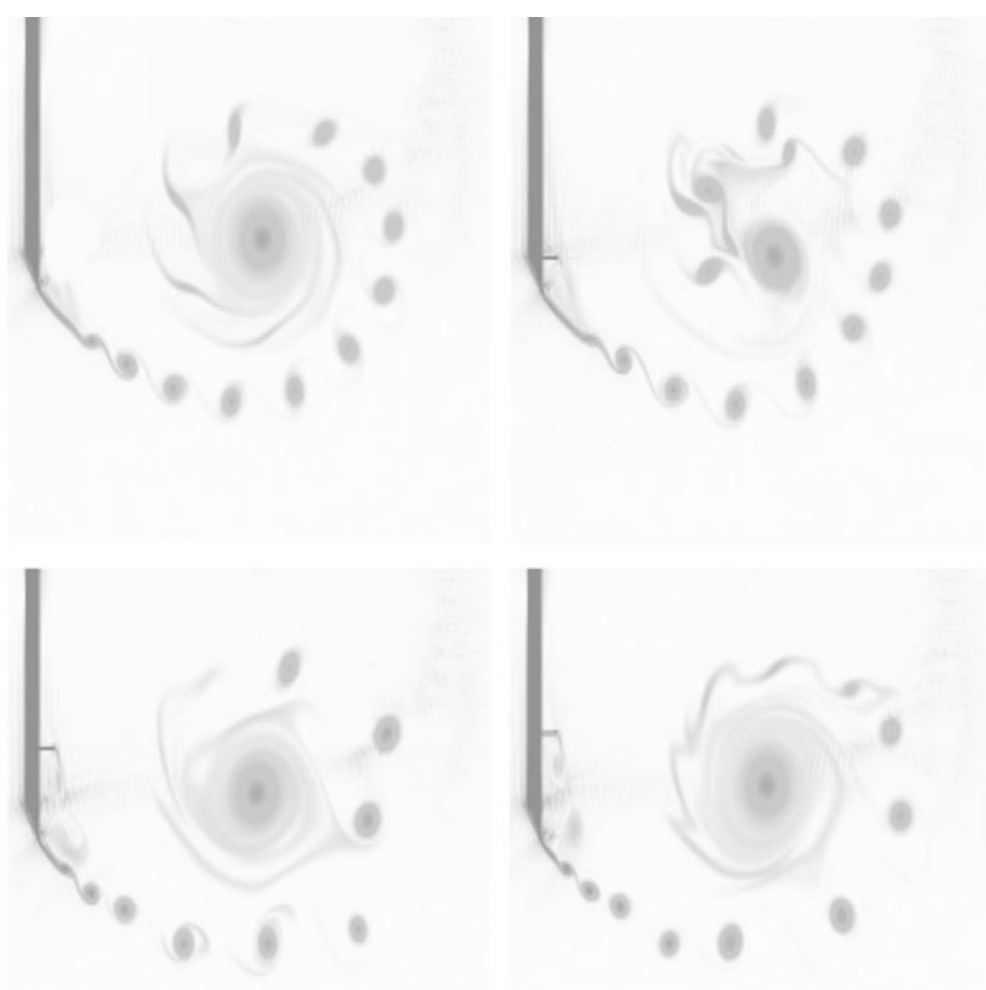

Fig. 10 Flow past an impulsively started plate with a sharp wedge, angle $\alpha=30^{\circ}$ at $R e=9500$. Vorticity fields at $t=2.01$ for a plate without riblet (top, left), and for plates with riblets at different positions 
$t=2.01$ without riblet (top, left) and for three different riblet positions. We observe that the dynamics of the secondary vortices are strongly modified by the riblet.

For increasing ratio $d / L$, we find that:

- the formation of the first secondary vortex is getting closer to the tip of the plate,

- the distance between two secondary vortices becomes irregular,

- therefore vortex pairings occur.

An interesting direction for future studies is the influence of the thickness of the plate and the role of the Reynolds number.

\section{Conclusion and perspectives}

We presented numerical simulations of two-dimensional incompressible viscous flows past a flat plates at $R e=9500$. A volume penalization approach is used to take into account the geometry of the plate with no-slip boundary conditions. We used two different numerical schemes, either a Fourier pseudospectral method, or an adaptive wavelet method, both at resolution $\left(\mathbf{N}=2048^{2}\right)$. Therewith, we have studied numerically the free shear layer instability of a flow past a flat plate for different shapes of the tips. We checked that the roll-up of the shear layer into a spiral, which forms the starting vortex, exhibits the scaling laws predicted by Saffman (1995) for both cases studied here, the impulsively started and the uniformly accelerated plate. At later times, we found that the shear layer becomes unstable and produces secondary vortices, as observed in both laboratory and numerical experiments. We also showed that the formation of the secondary vortices depends on the shape of the tips of the plate, and is enhanced by adding a riblet on its back. By varying the position and the size of the riblet, we tested that the dynamics of the secondary vortices can be strongly modified. The next step will be to develop systematic control strategies of the wake to enhance, but also to inhibit, the secondary vortices.

Acknowledgments We thank Thomas Leweke and Monika Nitsche for their fruitful discussions, and Dmitry Kolomenskiy for comments on the paper.

\section{References}

Abid M, Verga A (2002) Stability of a vortex sheet roll-up. Phys Fluids 14(11):3829-3834

Angot P, Bruneau C-H, Fabrie P (1999) A penalisation method to take into account obstacles in viscous flows. Num Math 81:497-520

Arquis E, Caltagirone JP (1984) Sur les conditions hydrodynamiques au voisinage d'une interface milieu fluide - milieux poreux: application à la convection naturelle. C R Acad Sci Paris II(299):1-4

Canuto C, Hussaini MY, Quateroni A, Zang TA (1988) Spectral methods in fluid dynamics. Springer, Berlin

Carbou G, Fabrie P (2003) Boundary layer for a penalization method for viscous incompressible flow. Adv Differ Equ 8:1453-1480

Farge M (1992) Wavelet transforms and their applications to turbulence. Ann Rev Fluid Mech 24:395-457

Farge M, Schneider K (2001) Coherent vortex simulation (CVS), a semi-deterministic turbulence model using wavelets. Flow Turbul Combust 66(4):393-426

Fröhlich J, Schneider K (1997) An adaptive wavelet-vaguelette algorithm for the solution of PDEs. J Comput Phys 130:174-190

Higuchi H, Balligand H, Strickland JH (1996) Numerical and experimental investigations of the flow over a disk undergoing unsteady motion. J Fluids Struct 10(7):705-719

Keetels GH, d'Ortona U, Kramer W, Clercx HJH, Schneider K, van Heijst GJF (2007) Fourier spectral and wavelet solvers for the incompressible Navier-Stokes equations with volume penalization: convergence of a dipole-wall collision. J Comput Phys 227:919-945 
Kevlahan N, Ghidaglia J-M (2001) Computation of turbulent flow past an array of cylinders using a spectral method with Brinkman penalization. Eur J Mech B 20:333-350

Khadra K, Parneix S, Angot P, Caltagirone J-P (2000) Fictious domain approach for numerical modelling of Navier-Stokes equations. Int J Num Meth Fluids 34:651-684

Kolomenskiy D, Schneider K (2009) A Fourier spectral method for the Navier-Stokes equations with volume penalisation for moving solid obstacles. J Comput Phys 228:5687-5709

Koumoutsakos P, Shiels D (1996) Simulations of the viscous flow normal to an impulsively started and uniformely accelerated flat plate. J Fluid Mech 328:177-227

Luchini P, Tognaccini R (1999) Comparison of viscous and inviscid numerical simulations of the start-up vortex issuing from a semi-infinite flat plate. ESAIM Proc 7:247-257

Luchini P, Tognaccini R (2002) The start-up vortex issuing from a semi-infinite flat plate. J Fluid Mech 455:175-193

Moore DW (1976) The stability of an evolving two-dimensional vortex sheet. Mathematica 23:35-44

Nitsche M (1996) Scaling properties of vortex ring formation at a circular tube opening. Phys Fluids 8(7):18481855

Peyret R (2002) Spectral methods for incompressible viscous flow, vol 148. Springer, Berlin

Pierce D (1961) Photographic evidence of the formation and growth of vorticity behind plates accelerated from rest in still air. J Fluid Mech 11:460-464

Prandtl L (1905) Über Flüssigkeitsbewegung bei sehr kleiner Reibung. Verhandlungen des III internationalen Mathematiker Kongresses (Heidelberg, 1904), Leipzig

Pullin DI (1978) The large scale structure of unsteady self-similar rolled-up vortex sheet. J Fluid Mech 88:401-430

Pullin DI, Perry AE (1980) Some flow visualization experiments on the starting vortex. J Fluid Mech 97:239255

Saffman PG (1995) Vortex dynamics. Cambridge University Press, Cambridge

Schneider K, Farge M (2002) Adaptive wavelet simulation of a flow around an impulsively started cylinder using penalisation. Appl Comput Harm Anal 12:374-380

Schneider K (2005) Numerical simulation of the transient flow behaviour in chemical reactors using a penalisation method. Comput Fluids 34(7):1223-1238

Schneider K, Farge M (2005) Numerical simulation of the transient flow behaviour in tube bundles using a volume penalisation method. J Fluids Struct 20(4):555-566

Schneider K, Vasilyev M (2010) Wavelet methods in computational fluid dynamics. Annu Rev Fluid Mech 42:473-503

Wang ZJ, Liu JG, Childress S (1999) Connection between corner vortices and shear layer instability in flow past an ellipse. Phys Fluids 11(9):2446-2448 